\section{Cell-cycle deregulation in progressive CML}

\section{Michael Savona and Moshe Talpaz}

There are alternative splice variants for CDKN2A and, in their correspondence on our Review (Savona, M. \& Talpaz, M. Getting to the stem of chronic myeloid leukemia. Nature Rev. Cancer 8, 341-350), Williams and Sherr correctly note that one of these variants is translated through an alternate reading frame (hence, $A R F$ ), which acts by stabilizing p53 protein levels by inhibiting MDM2-mediated ubiquination and degradation ${ }^{1,2}$. We apologize for oversimplifying this molecular biology; indeed, $A R F$ does not inhibit cyclin-dependent kinases (specifically, CDK4 and CDK6) as INK4A does, and we thank Williams and Sherr for clarifying this point. We failed to describe this important nuance in our efforts to express the known direct relationships between BCR-ABL and these tumour suppressors, their influence on G1 in the cell cycle, and their net pro-senescence effects. This was the planned design of the discussion on tumour suppressors in the latter half of our manuscript ${ }^{3}$.

Absence of the CDKN2A locus is a popular theme in liquid malignancies, and has been noted as a hallmark of aggressive disease in lymphocytic leukaemias for many years. ${ }^{4,5}$ At this point, it is still not clear whether the CDKN2A locus is deleted solely in lymphoid blast crisis, and not myeloid blast crisis chronic myeloid leukaemia (CML), as Williams and Sherr contend. There are early experimental data of loss of this locus also in myeloid malignancies ${ }^{6-8}$, and recent genomic analyses do not expressively indicate that this aberrancy is germane to lymphoid blast crisis, and exclusive of myeloid blast crisis or the rare entity Philadelphia chromosome $(\mathrm{Ph})^{+}$acute myeloid leukaemia (AML) ${ }^{9,10}$. That said, the authors have provided clear evidence that aberrancy of the CDKN2A locus is common and important in lymphoid leukaemias.
Likewise, we recognize the burgeoning data that the lack of the CDKN2A locus combined with BCR-ABL is sufficient to generate committed cells capable of self-renewal in a murine model of $\mathrm{Ph}^{+}$acute lymphocytic leukaemia (ALL) ${ }^{9,10}$. Though there is description of BCR-ABL-positivity and concomitant loss of $\mathrm{p} 16^{\mathrm{INK} 4 \mathrm{~A}}$ and $\mathrm{p} 14^{\mathrm{ARF}}$ in patients with $\mathrm{Ph}^{+}$acute leukaemias (and even some correlations with prognosis $)^{11}$, it has not yet been illustrated whether this interaction is direct, dependent and sequential in vivo, and not part of a cascade of progressive genetic instability. It is likely that a disruption of this and other cell cycle checkpoint kinases are part of many subsequent events that influence disease phenotype and progression. In the discussion of the carcinogenesis of CML, FIG. 3 of our paper ${ }^{3}$ was intended to, first, illustrate one example of an established tumour suppressor pathway(s) that BCR-ABL directly influences and, second, describe a known influence of epigenetic phenomena. In this light, data supporting the direct cause-andeffect relationship between $\mathrm{BCR}-\mathrm{ABL}$ and tumour suppressors from the CDKN2A locus as CML progresses are tenuous.

Finally, we reiterate the tenant of an anaplastic threshold (see FIG. 1 of the manuscript) ${ }^{3}$, which embodies our theory of carcinogenesis in CML: initially in the disease, and prior to the anaplastic threshold, BCR-ABL is a dominant oncogene on which the leukaemia cell depends for survival, and elimination of BCR-ABL - through imatinib, or other tyrosine kinase inhibitors - leads to remission; next, as the genetic instability grows, the disease approaches the anaplastic threshold - a period of transition at which the intracellular chaos induced by BCR-ABL begins to allow the leukaemic phenotype to be sustained irrespective of BCR-ABL; and, finally, there is a period of disease progression clearly beyond the anaplastic threshold in which BCR-ABL is superfluous and the leukaemic genotype can self-sustain the leukaemic phenotype. This leukaemic genotype is akin to that described in the recent genetic studies of advanced $\mathrm{CML}$ and $\mathrm{Ph}^{+} \mathrm{ALL}^{12,13}$. The difficulty of treating advanced CML lies in the fact that, at this point, BCR-ABL is just one member of an angry mob inciting anarchy, and not just a lone suppressible agitator.

University of Michigan, Internal Medicine Hematology Oncology, 1,500 East Medical Center Drive, Ann Arbor, Michigan 48109-5936, USA. Correspondence to M.S. and M.T. e-mails: msavona@med.umich.edu, mtalpaz@med.umich.edu

1. Bixby, D., Kujawski, L., Wang, S. \& Malek, S. N. The pre-clinical development of MDM2 inhibitors in chronic lymphocytic leukemia uncovers a central role for p53 status in sensitivity to Mdm2 inhibitormediated apoptosis. Cell Cycle 7, 971-979 (2008).

2. Williams, R. T. \& Sherr, C. J. BCR-ABL and CDKN2A: a dropped connection. Nature Rev. Cancer 8, 563 (2008).

3. Savona, M. \& Talpaz, M. Getting to the stem of chronic myeloid leukaemia. Nature Rev. Cancer 8, 341-350 (2008).

4. Heerema, N. A. et al. Secondary cytogenetic aberrations in childhood Philadelphia chromosome positive acute lymphoblastic leukemia are nonrandom and may be associated with outcome. Leukemia 18, 693-702 (2004).

5. Schoppmeyer, K., Norris, P. S. \& Haas, M. Inhibition of T-cell acute lymphoblastic leukemia proliferation in vivo by re-expression of the $\mathrm{p} 16^{\text {INK4a }}$ tumor suppressor gene. Neoplasia 1,128-137 (1999).

6. Ahuja, H., Bar-Eli, M., Advani, S. H., Benchimol, S. \& Cline, M. J. Alterations in the p53 gene and the clonal evolution of the blast crisis of chronic myelocytic leukemia. Proc. Natl Acad. Sci. USA 86, 6783-6787 (1989).

7. Calabretta, B., Perrotti, D. The biology of CML blast crisis. Blood 103, 4010-4022 (2004).

8. Feinstein, E. et al. p53 in chronic myelogenous leukemia in acute phase. Proc. Natl Acad. Sci. USA 88, 6293-6297 (1991).

9. Williams RT, den Besten, W. \& Sherr, C. J. Cytokinedependent imatinib resistance in mouse BCR-ABL ${ }^{+}$, Arf-null lymphoblastic leukemia. Genes Dev. 21 2283-2287 (2007).

10. Williams, R. T., Roussel, M. F. \& Sherr, C. J. Arf gene loss enhances oncogenicity and limits imatinib response in mouse models of $\mathrm{Bcr}-\mathrm{Abl}$-induced acute lymphoblastic leukemia. Proc. Natl Acad. Sci. USA 103, 6688-6693 (2006)

11. Mirebeau, D. et al. The prognostic significance of CDKN2A, CDKN2B and MTAP inactivation in B-lineage acute lymphoblastic leukemia of childhood. Results of the EORTC studies 58881 and 58951. Haematologica 91, 881-885 (2006).

12. Mullighan, C. G. et al. BCR-ABL1 lymphoblastic leukaemia is characterized by the deletion of Ikaros. Nature 453, 110-114 (2008).

13. Radich, J. P. et al. Gene expression changes associated with progression and response in chronic myeloid leukemia. Proc. Natl Acad. Sci. USA 8, 2794-2799 (2006). 